\title{
Predicción del rendimiento académico por medio de técnicas de inteligencia artificial
}

\author{
Omar D. Castrillón, William Sarache y Santiago Ruiz-Herrera \\ Universidad Nacional de Colombia - Sede Manizales, Facultad de Ingeniería y Arquitectura, Departamento de Ingeniería \\ Industrial, Grupo de Innovación y Desarrollo Tecnológico, Bloque Q Campus La Nubia, Manizales, 170001 - Colombia. \\ (e-mail: odcastrillong@unal.edu.co; wasarachec@unal.edu.co; sruizhe@unal.edu.co)
}

Recibido May. 9, 2019; Aceptado Jul. 8, 2019; Versión final Ago. 12, 2019, Publicado Feb. 2020

\begin{abstract}
Resumen
El objetivo de este artículo es predecir el rendimiento académico de estudiantes de educación superior, a partir de diversos factores influyentes usando técnicas de inteligencia artificial (clasificadores). El estudio de tales factores, aunque ha sido ampliamente analizado desde enfoques cuantitativos y cualitativos, aun presenta oportunidades de investigación usando herramientas que provee la inteligencia artificial, particularmente en la predicción del rendimiento académico. Con los factores definidos (educacionales, familiares, socioeconómicos, de hábitos y costumbres, entre otros), se diseñó una metodología que permite entrenar un sistema capaz de clasificar a priori a un nuevo estudiante, en una de las cinco categorías predeterminadas de rendimiento académico. Esta clasificación permite a una institución educativa identificar con anticipación estudiantes con problemas potenciales de rendimiento académico. A partir de ello, se pueden desplegar acciones de acompañamiento y mitigación inmediatas. La metodología fue aplicada a una muestra de estudiantes de una universidad pública en Colombia, obteniendo un nivel de aciertos del $91.7 \%$.
\end{abstract}

\section{Prediction of academic performance using artificial intelligence techniques}

\begin{abstract}
The aim of this article is to predict the academic performance of higher education students, considering several influential factors, applying artificial intelligence techniques (classifiers). Although such factors have been widely analyzed from quantitative and qualitative approaches, they still represent research opportunities using artificial intelligence tools, particularly in academic performance prediction. With the definition of influential factors (educational, family background, social and economics, habits and customs, among others), a methodology was designed to train a system able to a priori classify a new student, in one of the five categories of academic performance. This classification allows an educational institution to have an early identification of students with potential academic performance problems. From this knowledge the institution can deploy immediate mitigation action. The methodology was applied to a sample of students from a public university in Colombia, obtaining a success level of $91.7 \%$.
\end{abstract}




\section{INTRODUCCIÓN}

La búsqueda de la calidad en la educación es uno de los objetivos medulares de las instituciones académicas. Para tal fin se despliegan estrategias y programas que tienen como finalidad mejorar el desempeño y permanencia de los estudiantes (Helal et al, 2018). Sin embargo, definir la mezcla adecuada de acciones y decisiones estratégicas que maximicen el desempeño estudiantil no es una tarea fácil, dados los diferentes factores influyentes. El rendimiento académico de un estudiante se ha asociado a diversos factores personales, sociales e institucionales (Garbanzo, 2007; Secretaria de Educación Distrital de Bogotá, 2010). Para Rendón (1997), el éxito escolar está asociado a diferentes características, medios y valores. No obstante, la mayoría de los estudios relacionados con la predicción del rendimiento académico han estado asociados a la educación básica y primaria, siendo muy pocas las aplicaciones existentes en educación superior (Mandelman et al, 2016).

El rendimiento académico es una valoración de los logros obtenidos por el estudiante a lo largo del curso (https://definicion.de/rendimiento-academico/) . De acuerdo con la Secretaria de Educación Distrital de Bogotá (2010), esta valoración ha estado asociada a diversos factores tales como: estudiantes (hábitos de la persona como frecuencia de estudio y lectura, Tv, celular, trabajo), familia (entorno familiar, ingresos económicos, ambiente familiar), comunitarios (estrato socio-económico, deporte, aspectos positivos y negativos de la comunidad), universitarios (recursos, uso del tiempo libre en la universidad, relaciones con docentes y estudiantes, horarios, pedagogía de los profesores, etc.). Según Çilan y Can (2014), la mayoría de estos estudios se centran en señalar dificultades sin proponer soluciones reales.

Desde la perspectiva de las técnicas de inteligencia artificial, se identificaron diversos trabajos orientados a analizar el rendimiento de los estudiantes. En el estudio de Bravo et al. (2015), se aplicaron dos técnicas (bosques aleatorios y árboles de regresión), en una base de datos de estudiantes chilenos de octavo grado, con el fin de identificar y caracterizar los perfiles de los estudiantes con base al rendimiento académico obtenido en matemáticas. Se encontró que las expectativas educativas de los padres, el tipo de escuela y el índice de habilidades matemáticas, eran los factores de mayor influencia. En el trabajo de Miguéis et al. (2018) se usaron técnicas de minería de datos para clasificar y segmentar los estudiantes según su potencial académico con el fin de disminuir los fracasos, mejorar los resultados, conseguir más recursos. Con dicha técnica, los citados autores lograron un nivel de efectividad del $95 \%$.

Fernandes et al. (2019), usaron la técnica GBM (Gradient Boosting Machine), para predecir las variables académicas más influyentes en el rendimiento académico de un estudiante. Los resultados indican que el grado, el ausentismo, el barrio, la escuela y la edad, son los factores más influyentes. De-La-Hoz et al. (2019) se enfocaron en el manejo ambientes virtuales, con el fin de lograr una clasificación y predicción de usuarios según su conocimiento, utilizando un algoritmo de aprendizaje automático, basado en diversas técnicas (componentes principales, aprendizaje automático, clusterización, lógica difusa y $\mathrm{K}$ vecino más cercano, logrando una efectividad del 91\%. Así mismo, Taylan y Karagozog lu (2009) diseñaron un sistema basado en lógica difusa y redes neuronales con el objetivo de predecir el rendimiento académico de los estudiantes. En el trabajo de Hejazi et al. (2011), se usaron modelos para predecir el rendimiento académico en áreas como matemáticas a partir de variables tales como tareas motivadoras, objetivos, percepción de la instrumentalización y aprendizaje autorregulado.

En Hamsa et al. (2016), se combinaron algoritmos genéticos difusos y árboles de decisión, con el fin de predecir el rendimiento académico de estudiantes de pregrado y de maestría de los programas ciencias de la computación, electrónica y comunicaciones. Pandey y Taruna (2016) proponen un sistema de clasificadores múltiples integrados para predecir el rendimiento académico. Este sistema se basó en arboles de decisión, el k-ésimo vecino más cercano y estimadores de dependencia de agregación, lográndose una efectividad que fluctúa entre el $87 \%$ y el $99 \%$, según el tamaño de la base de datos empleada. En la publicación de Mitrofan y lon (2013), se analizan las relaciones existentes entre la personalidad y el rendimiento académico de un estudiante. El objetivo era predecir el rendimiento académico a partir de grandes factores de personalidad: Conciencia, auto disciplina, persistencia, auto organización y orientación al logro. En un trabajo similar, Paunonen y Ashton (2013) estudiaron la incidencia de la personalidad y la motivación en el rendimiento académico. Por su parte, otros autores como Etxeberria et al. (2017) relacionan el rendimiento académico con el perfil de ingreso de cada estudiante.

Por su parte, Vedel et al. (2015), analizaron los rasgos de personalidad, entre estudiantes de diferentes pregrados, frente al rendimiento académico de los mismos, encontrando diferencias significativas en cada uno de los grupos, con lo cual se pudo establecer una predicción acorde a tales rasgos. En general, las técnicas Inteligentes han sido empleadas para predecir una amplia variedad de eventos, entre los que se destacan: caracterización y predicción de descargas académicas (Li y Rijke, 2019); mejoramiento del rendimiento de equipos de trabajo en el aula (Alberola et al., 2016); predicción del rendimiento con base en 
factores cognitivos y no cognitivos (Fonteyne et al., 2017); sistemas basados en lógica difusa para medir el rendimiento de las universidades y su personal (Yousif y Shaout, 2018); empleo de sistemas de información geográfica para establecer la influencia de las zonas verdes en el rendimiento académico de un estudiante (Kweon et al., 2017); análisis del pensamiento crítico en el rendimiento académico de estudiantes de maestría (D’Alessio et al., 2019); ansiedad (Crişan y Copaci, 2015). Igualmente, se encontraron muy buenos estudios sobre el pronóstico de las acciones en la bolsa, realizados por personas, con un alto rendimiento académico (Zhu et al, 2018), aspecto que justifica más las investigaciones que se hagan en este campo.

Los diversos estudios realizados sobre rendimiento académico (Garbanzo, 2007; Secretaria de Educación Distrital de Bogotá, 2010), han identificado diversos factores y/o atributos influyentes en estudiantes de niveles de primaria y secundaria, con muy pocos avances en el ámbito de la formación superior, en donde otros factores pudiesen afectar el desempeño de los estudiantes. Lo anterior, genera oportunidades de investigación frente a la necesidad de establecer cuáles son los factores más predominantes en el rendimiento académico de un estudiante de pregrado, con el fin de proveer información de utilidad para desplegar una política académica en las diversas universidades, con miras a prevenir el fracaso estudiantil o mortalidad académica.

Desde esta perspectiva, el objetivo de este artículo es predecir el rendimiento académico de los estudiantes de pregrado que ingresan a una institución, usando técnicas de inteligencia artificial, basadas en clasificadores bayesianos, los cuales han tenido una amplia aplicación en muchos otros campos (Castrillón et al, 2017). Específicamente, el trabajo se llevó a cabo en una muestra de estudiantes de Ingeniería Industrial de una universidad pública en Colombia. Para tal fin, se consideraron un conjunto de factores ampliamente usados en la literatura tales como: género, edad, frecuencia de estudio, hábitos de lectura, uso de la televisión, uso del celular, trabajo, nivel escolaridad de los padres, elementos para estudiar en casa, nivel de ingresos, clima familiar, tiempo para estudiar en casa, estrato socio económico, actividades deportivas, actividades comunitarias, otras actividades, recursos universitarios, infraestructura, manejo del tiempo en la universidad, relaciones con los profesores, calidad de los profesores, horarios de clase y pedagogía (Secretaria de Educación Distrital de Bogotá, 2010; Castrillón et al, 2018).

\section{MATERIALES Y MÉTODOS}

El desarrollo de la metodología se hizo por medio de técnicas de clasificación inteligente, basados en algoritmos bayesianos y otros, ejecutados mediante el programa Weka (https://www.cs.waikato.ac.nz/ml/weka/). Estas técnicas, de forma a priori, permiten predecir el desempeño académico de un estudiante que ingresa a la universidad y, a partir de ello, establecer estrategias más personalizadas, programas de ayuda y de seguimiento, en aquellos estudiantes cuyo rendimiento proyectado arroje resultados no satisfactorios. De esta manera, también es posible determinar los factores incidentes en el rendimiento académico de un estudiante con miras a desplegar programas de mejora continua a nivel institucional. La metodología empleada en el estudio se expone de manera resumida en los siguientes ocho pasos: (1) Diseño de la encuesta y recolección de la información; (2) Definición de la muestra; (3) Construcción de la base de datos; (4) Correlación entre atributos; (5) Procesamiento computacional; (6) Predicción del rendimiento académico; (7) Identificación de los principales atributos influyentes en el desempeño académico; y (8), Estabilidad del sistema.

Paso 1: Diseño de la encuesta y recolección de la información. Con el fin de analizar cada uno de los 22 atributos intervinientes en el estudio de clasificación, estos fueron agrupados en 4 factores: Factor 1 (F1): estudiantes; Factor 2 (F2): familia; Factor 3 (F3): aspectos comunitarios y Factor 4 (F4): aspectos universitarios. Esta agrupación se realizó tomando como referencia los lineamientos emitidos por la Secretaria de Educación Distrital (Secretaria de Educación Distrital de Bogotá, 2010). Estos factores fueron evaluados usando una escala de cinco niveles: 1: nunca; 2: rara vez; 3: algunas veces; 4: casi siempre; 5: siempre. No obstante, en algunas preguntas debe entenderse como 1 el nivel más bajo, pésimo o ninguno y 5 el nivel más alto, muchos o excelente.

Paso 2. Definición de la muestra. Con el fin de obtener una mayor confiabilidad estadística se aplicó un muestreo aleatorio simple, sobre 460 estudiantes pertenecientes al programa de pregrado objeto de estudio. Este cálculo de la muestra se realizó por medio de la Ecuación (1) (Valdivieso et al., 2011).

$$
\mathrm{n}=\frac{\mathrm{I}^{2} \mathrm{pqM}}{\mathrm{ME}^{2}+\mathrm{I}^{2} \mathrm{pq}}
$$

Dónde: n: dimensión de la muestra. I: Intervalo de confianza, obtenido por medio de una distribución normal estándar invertida. $\mathrm{p}$ y q: representan las variaciones éxito y fracaso, respectivamente. M: Número de estudiantes del programa de pregrado. E: Error muestral. 
Paso 3: Construcción de la base de datos. La encuesta se aplicó a un número n (obtenido de la ecuación 1) de individuos seleccionados al azar, lo cual se considera adecuado cuando se trata de aplicar modelos bayesianos (Valencia et al., 2015). Una vez recolectadas y tabuladas las encuestas, estas fueron organizadas en 5 grupos según el promedio académico de cada estudiante: A: Promedio entre [4.6 - 5.0]; B: promedio entre [4.2 - 4.6); C: promedio entre [3.8 - 4.2); D: promedio entre [3.4 - 3.8); E: promedio entre [0.0 - 3.4). Se aclara que en la muestra no se encontraron estudiantes con un promedio inferior a 3.0.

Paso 4: Correlación entre atributos. En este punto se construye una matriz de correlación de los 22 atributos analizados, respecto a la variable dependiente denominada "Prom". Esta correlación permitirá: a) demostrar que el efecto producido sobre la variable dependiente no es producto de una única variable independiente y b) establecer las variables que presentan una mayor correlación con la variable "Prom" y realizar así una selección previa de variables, antes de emplear el clasificador bayesiano por medio del cual se realiza el respectivo análisis multivariante.

Paso 5: Procesamiento computacional. Con base en los grupos antes mencionados, y en los atributos seleccionados en el paso anterior, se diseñó el encabezado del archivo Arff para la plataforma de software libre distribuido bajo la licencia GNU-GPL, el cual permite aprendizaje automático y minería de datos (https://es.wikipedia.org/wiki/Weka_(aprendizaje_autom\%C3\%A1tico)). Esta herramienta informática, denominada WEKA, fue desarrollada en la Universidad de Waikato (https://www.cs.waikato.ac.nz/ml/weka/). Este encabezado contempla cada una de los 22 atributos analizadas en la encuesta, más el atributo promedio de cada estudiante. Adicionalmente, con los resultados obtenidos en el paso 2, se estructuró el cuerpo del archivo. Arff para ser analizado desde el programa Weka, por medio de las diferentes técnicas de inteligencia artificial ofrecidas por esta herramienta computacional.

Paso 6: Predicción del rendimiento académico. El archivo diseñado y construido en el paso anterior, fue analizado usando un clasificador Bayesiano. Si bien otras técnicas pueden arrojar buenos resultados, la principal ventaja del clasificador bayesiano es que permite obtener muy buenos resultados con pocos datos (Valencia et al., 2015). No obstante, en este análisis se realizó con un tamaño de muestra valido (Ecuacion1).

Paso 7: Identificación de los principales atributos influyentes en el desempeño académico. Por medio del algoritmo de clasificación bayesiano J48, el software weka permitió obtener el respectivo árbol de decisión (http://weka.sourceforge.net/doc.dev/weka/classifiers/trees/J48.html). Con base en este árbol, se pudieron identificar los principales atributos influyentes en rendimiento académico de los estudiantes.

Paso 8. Estabilidad del sistema. Las soluciones generadas son repetidas durante una determinada cantidad de veces ó tratamientos. Con los mejores resultados en cada tratamiento, se realiza un análisis de varianza bajo el modelo $y_{i}=\mu+T_{i}+\varepsilon i$, donde $y_{i}$ representa la variable de la respuesta, $T_{i}$, el efecto causado por $i$ th tratamiento, $\varepsilon i$, el ith error experimental. La información recolectada debe cumplir las condiciones de independencia y normalidad requeridas.

\section{RESULTADOS}

Al aplicar la metodología antes descrita se obtuvo, en el Paso 1, la encuesta cuya estructura se muestra en la Tabla 1 ( 1 el nivel más bajo o pésimo y 5 el nivel más alto o excelente).

Tabla 1: Factores analizados. Fuente: Adaptado de Castrillón et al. (2018)

\begin{tabular}{|c|c|c|c|c|c|c|}
\hline Factor & Atributos & $\begin{array}{l}\text { Nunca } \\
\text { (1) }\end{array}$ & $\begin{array}{c}\text { Rara Vez } \\
\text { (2) }\end{array}$ & Algunas veces (3) & $\begin{array}{c}\text { Casi Siempre } \\
\text { (4) }\end{array}$ & $\begin{array}{c}\text { Siempre } \\
\text { (5) }\end{array}$ \\
\hline \multirow{5}{*}{ F1 } & Frecuencia de estudio (c) & & & & & \\
\hline & Frecuencia de lectura (d) & & & & & \\
\hline & Frecuencia de uso del tv (e) & & & & & \\
\hline & Frecuencia de uso celular (f) & & & & & \\
\hline & Frecuencia de trabajo $(\mathrm{g})$ & & & & & \\
\hline \multirow{5}{*}{ F2 } & Nivel escolar padres (a) & & & & & \\
\hline & Elementos para estudiar en casa (b) & & & & & \\
\hline & Nivel Ingresos (c) & & & & & \\
\hline & Clima Familiar (d) & & & & & \\
\hline & Tiempo para estudiar casa (e) & & & & & \\
\hline \multirow{5}{*}{ F3 } & Estrato Socioeconómico (a) & & & & & \\
\hline & Actividades deportivas (b) & & & & & \\
\hline & Actividades Comunitarias (c) & & & & & \\
\hline & Otras actividades positivas(d) & & & & & \\
\hline & Otras actividades negativas (e) & & & & & \\
\hline
\end{tabular}


Tabla 1. Continuación.

\begin{tabular}{|c|c|c|c|c|c|c|}
\hline Factor & Atributos & $\begin{array}{c}\text { Nunca } \\
\text { (1) }\end{array}$ & $\begin{array}{c}\text { Rara Vez } \\
\text { (2) }\end{array}$ & Algunas veces (3) & $\begin{array}{c}\text { Casi Siempre } \\
\text { (4) }\end{array}$ & $\begin{array}{l}\text { Siempre } \\
\text { (5) }\end{array}$ \\
\hline \multirow{7}{*}{ F4 } & Recursos (a) & & & & & \\
\hline & Infraestructura (b) & & & & & \\
\hline & Uso tiempo universidad (c) & & & & & \\
\hline & Relaciones Docentes (d) & & & & & \\
\hline & Docentes (e) & & & & & \\
\hline & Horarios (f) & & & & & \\
\hline & Pedagogía (g) & & & & & \\
\hline
\end{tabular}

Paso 2. Definición de la muestra. Con base en la ecuación (1), se calculó el tamaño de la muestra con los siguientes parámetros: $M=460$ estudiantes; $I=1,2815$. Intervalo de confianza, obtenido por medio de una Distribución normal estándar Invertida. Se obtiene con la función de Excel denominada inv.norm.estand (0.9); $p=0,5 ; q=0,5 ; E=0,05$ (error muestral del $5 \%$, para un intervalo del $90 \%$ ). El tamaño de muestra obtenido (n) fue de 121.

Paso 3 - 4. Construcción de la base de datos y Correlación entre atributos. Con base en los resultados del paso anterior, se realizaron 121 encuestas. La Tabla 2 expone, a manera de ejemplo, una parte de la base de datos para los primeros 22 estudiantes.

Tabla 2: Ejemplo base de datos inicial

\begin{tabular}{|c|c|c|c|c|c|c|c|c|c|c|c|c|c|c|c|c|c|c|c|c|c|c|}
\hline \multicolumn{1}{|c|}{$\mathrm{F} 1$} & \multicolumn{1}{|c|}{$\mathrm{F} 2$} & \multicolumn{3}{|c|}{$\mathrm{F} 3$} & \multicolumn{1}{c|}{ Prom } \\
\hline $\mathrm{c}$ & $\mathrm{d}$ & $\mathrm{e}$ & $\mathrm{f}$ & $\mathrm{g}$ & $\mathrm{a}$ & $\mathrm{b}$ & $\mathrm{c}$ & $\mathrm{d}$ & $\mathrm{e}$ & $\mathrm{a}$ & $\mathrm{b}$ & $\mathrm{c}$ & $\mathrm{d}$ & $\mathrm{e}$ & $\mathrm{a}$ & $\mathrm{b}$ & $\mathrm{c}$ & $\mathrm{d}$ & $\mathrm{e}$ & $\mathrm{f}$ & $\mathrm{g}$ & \\
\hline 3 & 3 & 3 & 5 & 1 & 5 & 5 & 5 & 5 & 4 & 3 & 3 & 4 & 4 & 2 & 4 & 5 & 4 & 4 & 5 & 5 & 4 & $\mathrm{~A}$ \\
\hline 4 & 3 & 2 & 3 & 2 & 1 & 3 & 1 & 4 & 3 & 3 & 1 & 1 & 3 & 1 & 2 & 5 & 3 & 4 & 4 & 4 & 4 & $\mathrm{~B}$ \\
\hline 4 & 4 & 2 & 2 & 2 & 3 & 3 & 2 & 3 & 4 & 2 & 3 & 3 & 3 & 3 & 3 & 4 & 3 & 4 & 4 & 4 & 4 & $\mathrm{~B}$ \\
\hline 3 & 2 & 2 & 4 & 2 & 3 & 4 & 3 & 4 & 3 & 3 & 5 & 2 & 4 & 2 & 4 & 3 & 3 & 5 & 4 & 4 & 4 & $\mathrm{~B}$ \\
\hline 2 & 3 & 4 & 4 & 3 & 4 & 4 & 3 & 3 & 4 & 3 & 2 & 2 & 2 & 3 & 4 & 4 & 3 & 4 & 5 & 4 & 4 & $\mathrm{~B}$ \\
\hline 4 & 2 & 2 & 4 & 1 & 4 & 4 & 4 & 4 & 4 & 3 & 3 & 2 & 4 & 2 & 3 & 5 & 4 & 4 & 4 & 3 & 5 & $\mathrm{C}$ \\
\hline 3 & 3 & 4 & 4 & 1 & 1 & 4 & 4 & 4 & 4 & 4 & 4 & 3 & 4 & 2 & 4 & 5 & 4 & 4 & 4 & 4 & 4 & $\mathrm{C}$ \\
\hline 3 & 3 & 2 & 4 & 2 & 3 & 3 & 3 & 4 & 4 & 3 & 3 & 3 & 3 & 2 & 3 & 4 & 4 & 4 & 4 & 3 & 4 & $\mathrm{C}$ \\
\hline 3 & 3 & 2 & 4 & 1 & 4 & 3 & 3 & 4 & 4 & 2 & 3 & 2 & 2 & 3 & 3 & 4 & 4 & 4 & 4 & 4 & 4 & $\mathrm{C}$ \\
\hline 4 & 4 & 2 & 3 & 1 & 1 & 3 & 2 & 4 & 4 & 1 & 4 & 3 & 4 & 1 & 4 & 4 & 4 & 4 & 4 & 4 & 4 & $\mathrm{C}$ \\
\hline 4 & 3 & 4 & 5 & 3 & 1 & 5 & 3 & 5 & 4 & 3 & 4 & 2 & 3 & 1 & 5 & 5 & 4 & 3 & 3 & 3 & 4 & $\mathrm{C}$ \\
\hline 4 & 5 & 2 & 5 & 1 & 1 & 5 & 3 & 4 & 4 & 3 & 5 & 1 & 2 & 3 & 5 & 5 & 4 & 4 & 4 & 4 & 4 & $\mathrm{C}$ \\
\hline 5 & 5 & 4 & 4 & 3 & 5 & 5 & 4 & 5 & 4 & 5 & 1 & 1 & 1 & 1 & 4 & 5 & 4 & 4 & 4 & 4 & 4 & $\mathrm{C}$ \\
\hline 4 & 3 & 2 & 4 & 1 & 1 & 1 & 3 & 5 & 4 & 2 & 4 & 2 & 3 & 1 & 4 & 5 & 4 & 3 & 4 & 4 & 5 & $\mathrm{C}$ \\
\hline 3 & 3 & 1 & 5 & 1 & 1 & 4 & 3 & 3 & 4 & 3 & 3 & 1 & 3 & 1 & 4 & 4 & 4 & 4 & 4 & 4 & 4 & $\mathrm{C}$ \\
\hline 4 & 4 & 1 & 5 & 1 & 1 & 2 & 3 & 4 & 4 & 3 & 2 & 1 & 4 & 3 & 2 & 5 & 5 & 4 & 1 & 3 & 4 & $\mathrm{D}$ \\
\hline 5 & 3 & 5 & 5 & 1 & 5 & 5 & 3 & 5 & 5 & 4 & 4 & 3 & 3 & 1 & 4 & 5 & 5 & 5 & 5 & 5 & 5 & $\mathrm{D}$ \\
\hline 4 & 1 & 5 & 5 & 1 & 5 & 5 & 5 & 5 & 5 & 5 & 4 & 1 & 3 & 2 & 5 & 5 & 5 & 4 & 5 & 4 & 5 & $\mathrm{D}$ \\
\hline 3 & 2 & 2 & 3 & 1 & 1 & 4 & 1 & 3 & 4 & 1 & 3 & 3 & 4 & 2 & 1 & 4 & 4 & 3 & 4 & 3 & 4 & $\mathrm{D}$ \\
\hline 3 & 3 & 4 & 4 & 1 & 1 & 4 & 3 & 4 & 5 & 3 & 1 & 3 & 4 & 1 & 4 & 5 & 5 & 3 & 3 & 4 & 4 & $\mathrm{D}$ \\
\hline 3 & 3 & 2 & 5 & 1 & 1 & 4 & 3 & 4 & 3 & 3 & 4 & 1 & 2 & 1 & 5 & 5 & 4 & 5 & 5 & 4 & 4 & $\mathrm{D}$ \\
\hline 4 & 3 & 2 & 5 & 2 & 3 & 4 & 3 & 4 & 5 & 3 & 5 & 3 & 4 & 2 & 4 & 4 & 4 & 3 & 4 & 4 & 4 & $\mathrm{D}$ \\
\hline
\end{tabular}

En esta tabla se calculó la correlación de cada uno de las 22 columnas iniciales (variables independientes o atributos) respecto a la última columna denominada Prom (Variable dependiente). Para el cálculo de esta correlación los valores de la columna "Prom", fueron sustituidos de la siguiente forma A $\rightarrow 5, B \rightarrow 4, C \rightarrow 3, D$ $\rightarrow 2$, E $\rightarrow 1$. La Tabla 3 expone los resultados obtenidos.

Tabla 3: Correlación variables independientes (Atributos) respecto a la variable dependiente (Prom)

\begin{tabular}{|c|c|c|c|c|c|c|c|c|c|c|c|c|}
\hline Correlación & \multicolumn{5}{|c|}{ F1 } & \multicolumn{7}{|c|}{ F2 } \\
\hline & c & $d$ & $\mathrm{e}$ & $f$ & $g$ & $a$ & $b$ & c & $d$ & & $\mathrm{e}$ & \\
\hline Prom & 0,14 & 0,12 & 0,04 & $-0,22$ & 0,40 & 0,10 & 0,03 & 0,02 & 0,17 & & 0,12 & \\
\hline Correlación & \multicolumn{5}{|c|}{ F3 } & \multicolumn{7}{|c|}{ F4 } \\
\hline & A & $b$ & c & $\mathrm{d}$ & $\mathrm{e}$ & a & $b$ & C & d & $\mathrm{e}$ & $f$ & $\mathrm{~g}$ \\
\hline Prom & $-0,02$ & 0,03 & 0,19 & 0,13 & $-0,13$ & $-0,08$ & $-0,04$ & $-0,03$ & 0,31 & 0,39 & 0,50 & 0,46 \\
\hline
\end{tabular}


Los resultados de la Tabla 3 indican que la variable dependiente no es el resultado de una sola variable independiente. Por otro lado, las variables que correlacionan significativamente (variables sombreadas), fueron las siguientes: F1g (Frecuencia de trabajo), F4d (relaciones docentes), F4e (Docentes), F4f (Horarios), F4g (Pedagogía).

Paso 5: Procesamiento computacional. Tomando como referencias las variables seleccionadas en el paso anterior, se escribió el archivo.Arff, para ser interpretado por la plataforma Weka. Este archivo se construyó con base en los atributos F1g, F4d, F4e, F4f, F4g y el atributo Prom, el cual ilustra cada una de las cinco categorías en que fue clasificado el promedio de los estudiantes. Los atributos F1g, F4d, F4e, F4f, F4g fueron definidos numéricamente en el encabezado del archivo.Arff , mientras que el atributo Prom fue definido de la siguiente forma: Prom \{"A","B","C","D","E"\}. A manera de ilustración, en la Tabla 4, se muestra una parte de los datos del mencionado archivo.

Tabla 4. Datos del archivo .Arff creado.

\begin{tabular}{|c|c|c|c|c|c|}
\hline F1g & F4d & F4e & F4f & F4g & Prom \\
\hline 1 & 4 & 5 & 5 & 5 & A \\
\hline 2 & 4 & 4 & 4 & 4 & B \\
\hline 2 & 4 & 4 & 4 & 4 & B \\
\hline 2 & 5 & 4 & 4 & 5 & B \\
\hline 3 & 4 & 5 & 4 & 4 & B \\
\hline 1 & 4 & 4 & 3 & 5 & C \\
\hline 1 & 4 & 4 & 4 & 4 & C \\
\hline 2 & 4 & 4 & 3 & 4 & C \\
\hline 1 & 4 & 4 & 4 & 4 & C \\
\hline 1 & 4 & 4 & 4 & 4 & C \\
\hline 3 & 3 & 3 & 3 & 4 & C \\
\hline 1 & 4 & 4 & 4 & 4 & C \\
\hline 1 & 4 & 5 & 4 & 5 & D \\
\hline 1 & 3 & 4 & 3 & 4 & D \\
\hline 1 & 3 & 3 & 4 & 4 & D \\
\hline 1 & 3 & 3 & 4 & 3 & D \\
\hline 1 & 5 & 5 & 4 & 4 & D \\
\hline 1 & 2 & 1 & 3 & 1 & E \\
\hline 1 & 4 & 4 & 2 & 3 & E \\
\hline 2 & 3 & 3 & 3 & 3 & E \\
\hline
\end{tabular}

Paso 6 -7: Predicción del rendimiento académico e Identificación de los principales atributos influyentes en el desempeño académico. En este paso, mediante el algoritmo J48 de la plataforma Weka (http://weka.sourceforge.net/doc.dev/weka/classifiers/trees/J48.html), se obtuvo un nivel de acierto del $91.67 \%$ en la predicción del rendimiento académico (ver tabla 5).

Tabla 5. Aciertos encontrados. Porcentajes de aciertos con diferentes tratamientos.

\begin{tabular}{|l|c|c|c|c|c|}
\hline \multicolumn{1}{|c|}{ Técnica empleada } & \multicolumn{5}{|c|}{ Porcentaje de aciertos (\%) } \\
\hline \multicolumn{1}{|c|}{} & Replica 1 & Replica 2 & Replica 3 & Replica 4 & Replica 5 \\
\hline $\begin{array}{l}\text { Validación Cruzada. Entrenamiento (50\%) y Validación } \\
(50 \%)\end{array}$ & 91.67 & 91.67 & 91.67 & 91.67 & 91.67 \\
\hline $\begin{array}{l}\text { Partición de la Base de datos. Entrenamiento (60\%) y } \\
\text { Validación (40\%) }\end{array}$ & 91.67 & 91.67 & 91.67 & 91.67 & 91.67 \\
\hline $\begin{array}{l}\text { Partición de la Base de datos. Entrenamiento (70\%) y } \\
\text { Validación (30\%) }\end{array}$ & 91.67 & 91.67 & 91.67 & 91.67 & 91.67 \\
\hline $\begin{array}{l}\text { Partición de la Base de datos. Entrenamiento (80\%) y } \\
\text { Validación (20\%) }\end{array}$ & 91.67 & 91.67 & 91.67 & 91.67 & 91.67 \\
\hline
\end{tabular}

Adicionalmente, en este punto se identificaron los principales factores influyentes en el desempeño académico y se obtuvo el árbol de clasificación usando la plataforma Weka (ver Figura 1) a partir del algoritmo J48 (Tabla 5. En este árbol es posible identificar las ramas de atributos que conducen a los mayores promedios académicos ( $A$ y B). De esta misma forma, se pueden encontrar las ramas conducentes al peor promedio (E). Un análisis de la Figura 1, permite identificar las ramas que llegan a los nodos finales (hojas), cada una de los cuales representa el promedio académico de los estudiantes según los grupos en que fueron clasificados 
A, B, C, D, E. La secuencia de pasos desde el nodo inicial hasta el nodo final (hojas) representa los atributos conducentes a obtener un mejor promedio académico. Los promedios académicos $A, B$ y $E$ son obtenidos según la siguiente secuencia de atributos: $\mathrm{F} 4 \mathrm{~g}$ (buena pedagogía) $\rightarrow \mathrm{F} 4 \mathrm{f}$ (buenos horarios) $\rightarrow \mathrm{F} 4 \mathrm{~d}$ (buenas relaciones docentes ) $\rightarrow$ A, F4g (buena pedagogía) $\rightarrow$ F4f (buenos horarios) F1g (trabajo remunerado) $\rightarrow$ F4d (buenas relaciones docentes) $\rightarrow \mathrm{B}, \quad \mathrm{F} 4 \mathrm{~g}$ (buena pedagogía) $\rightarrow$ F4f (buenos horarios) F1g (trabajo remunerado) $\rightarrow \mathrm{F} 4 \mathrm{e}$ (excelentes docentes) $\rightarrow \mathrm{B}, \mathrm{F} 4 \mathrm{~g}$ (mala pedagogía) $\rightarrow \mathrm{F} 4 \mathrm{f}$ (malos horarios) $\rightarrow \mathrm{E}$.

De otro lado, un análisis de la Figura 1 muestra que cuando hay exceso de confianza en la relaciones profesorestudiante, esto puede incidir negativamente en el rendimiento académico: F4g (Buena pedagogía) $\rightarrow$ F4f (Buenos Horarios) $\rightarrow$ F4d (Docentes (Excesos)) $\rightarrow$ D. Así, mismo si se tiene una buena pedagogía, pero las clases se imparten en horarios inadecuados, y los estudiantes no trabajan, se producen rendimientos académicos regulares o malos, es decir C y D. Esto es: F4g (buena pedagogía) $\rightarrow$ F4f (malos horarios) $\rightarrow$ F1g (No trabajo) $\rightarrow$ C ó D.

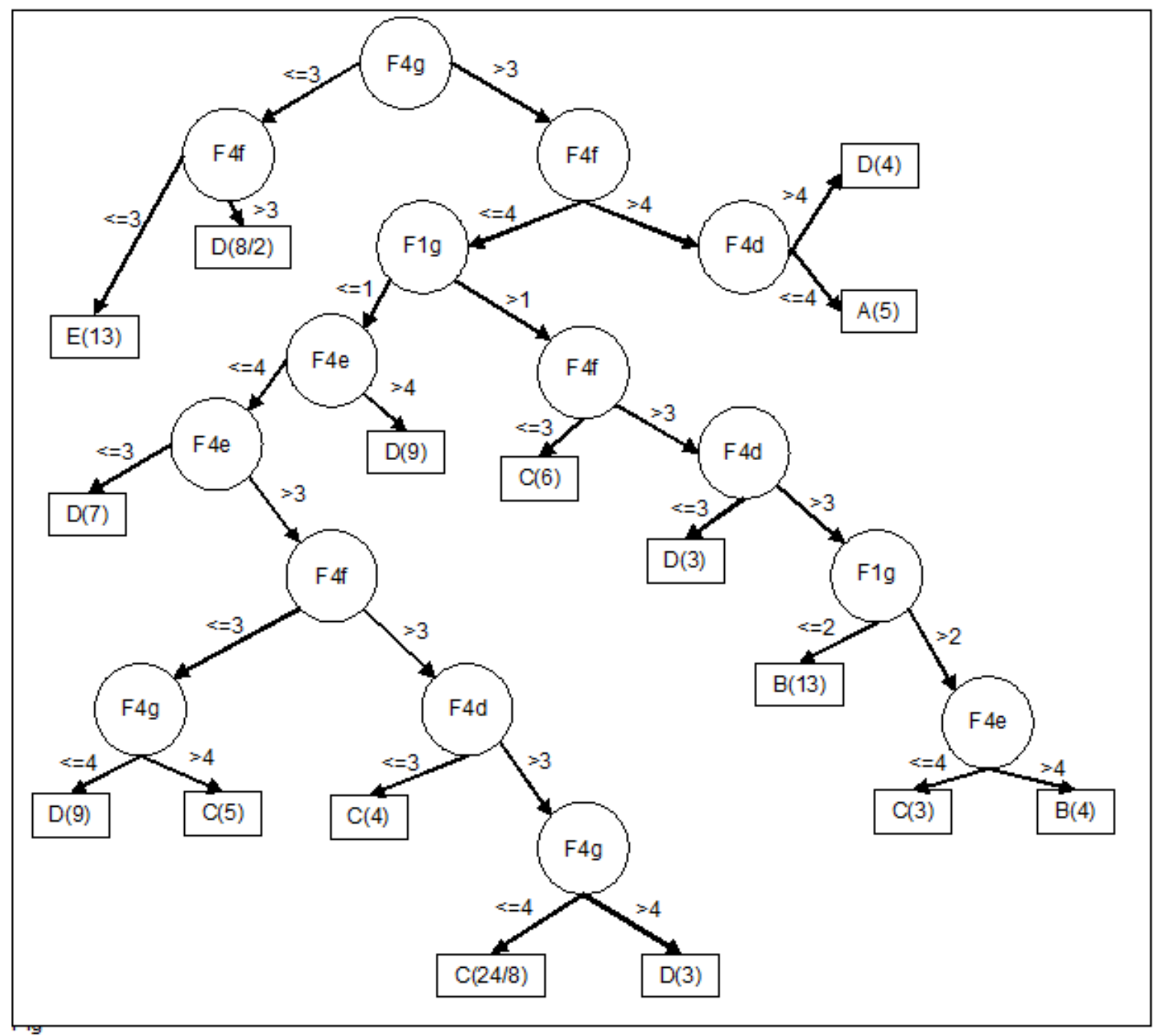

Fig. 1: Árbol de decisión generado por el programa Weka (https://www.cs.waikato.ac.nz/ml/weka/)

Paso 8. Estabilidad del sistema. Tomando como referencia la tabla 5, se llevó a cabo el análisis de ANOVA que se muestra en la Tabla 6.

Tabla 6. Análisis ANOVA.

\begin{tabular}{|l|r|r|r|r|r|}
\hline Fuente Variación & $\begin{array}{c}\text { Grados } \\
\text { Libertad }\end{array}$ & $\begin{array}{c}\text { Suma } \\
\text { Cuadrados }\end{array}$ & $\begin{array}{c}\text { Media } \\
\text { Cuadrática }\end{array}$ & $F_{\text {cal }}$ & F Tab \\
\hline Sum C Total & & 0,00 & & & \\
\hline Tratamiento & 3,00 & 0,00 & 0,00 & 1,00 & 2,87 \\
\hline Error Experimental & 36,00 & 0,00 & 0,00 & & \\
\hline Total & 39,00 & 0,00 & 0,00 & & \\
\hline El modelo no es significativo & & & & & \\
\hline
\end{tabular}


Dado que en el modelo el $\mathrm{F}_{\text {calculado }}$ es menor que el $\mathrm{F}_{\text {tabulado, }}$, se puede concluir que el modelo no es significativo. Es decir, bajo un intervalo de confianza del 90\%, los datos son estadísticamente iguales. Dado que cada tratamiento empleado en la tabla 5 siempre generó el mismo resultado, solo se calcularon 5 réplicas para cada uno de los tratamientos empleados.

\section{DISCUSIÓN}

Mediante el proceso de clasificación basado en técnicas inteligentes, se identificaron los atributos más influyentes en el rendimiento académico. El trabajo realizado muestra que, un adecuado rendimiento académico parte de una buena pedagogía, acompañada de buenos horarios y buenas relaciones con los docentes sin llegar a excesos. Existen otros factores importantes en el rendimiento académico como la calidad de los docentes, siendo además muy importante que los estudiantes trabajen un poco o realicen prácticas remuneradas, pues este tipo de actividades extracurriculares es formativo para la persona. No obstante, en la revisión de literatura (referencias), se pueden identificar una gran cantidad de categorías o grupos de factores influyentes en el rendimiento académico: Económicos, sociales, demográficos, familiares, personales, institucionales. Si bien, no todos los anteriores factores (grupos de atributos) pueden ser controlados por las instituciones educativas, este trabajo muestra que, si la institución se centra en controlar los 5 atributos identificados en esta investigación (pedagogía, horarios, relaciones docentes vs estudiantes, calidad de los docentes y además procurar por que los estudiantes tengan experiencia de prácticas en empresas), se puede influir notoriamente en el rendimiento académico de un estudiante.

Aunque este estudio solo se realiza sobre un programa de pregrado específico, los resultados muestran que las técnicas inteligentes y, en especial, las técnicas bayesianas, permiten obtener muy buenos resultados con un conjunto pequeños de datos (es decir, aunque la muestra obtenida se pequeña los resultados son fiables) (Valencia et al., 2015). No obstante, se parte de una muestra estadísticamente valida. Los resultados encontrados en este trabajo permiten inferir que un excelente o muy buen promedio académico depende de la dedicación y empeño que los docentes y estudiantes pongan en sus clases, más que de los mismos recursos materiales con que cuente la institución. La buena interacción de los anteriores factores permitirá un buen promedio académico. No obstante, aunque se logre lo anterior, si el trabajo de los estudiantes decae o si algunos de los demás factores analizados bajan considerablemente, el promedio también podrá bajar notoriamente e incluso podría llegar a ser malo. En síntesis, según los resultados obtenidos, en la muestra de estudiantes analizada, el rendimiento académico está más ligado a factores humanos y no a los recursos materiales.

Si bien existen diversos trabajos sobre este tema, los cuales asocian el rendimiento académico a factores personales, sociales, institucionales, recursos, valores, entusiasmo, grado escolar, tipo de escuela, edad, zonas verdes, etc., este trabajo asocia el rendimiento académico específicamente a factores humanos relacionados con los docentes y estudiantes; factores en los cuales se deberían centrar las instituciones educativas, como se expresó en el párrafo anterior. Es importante volver a resaltar que las relaciones docentes tienen un papel muy importante en el rendimiento académico, jugando también un papel muy importante el hecho de que los estudiantes puedan realizar pequeñas prácticas (como ya se expresó), un aspecto que termina siendo formativo para el estudiante y puede ayudarle a manejar mejor su tiempo y organizarse frente a los horarios universitarios. Sin embargo, un tiempo alto de trabajo, puede generar efectos negativos.

Al comparar los porcentajes de clasificación (respecto a la predicción del rendimiento académico) obtenidos en este estudio (ver Tabla 5), con los porcentajes de clasificación obtenidos por algunos trabajos citados en la introducción de este artículo, se encuentra que el nivel de acierto es alto, lo cual respalda la fiabilidad al estudio. Sin embargo, es importante resaltar que los estudios realizados son válidos para el contexto en el cual se aplicó, pudiendo diferir entre diferentes carreras profesionales, niveles de formación (pregradomaestría-doctorado), regiones, ciudades o países. No obstante, la metodología puede ser replicada en toda una región o inclusive a nivel de un país, e incluir otros factores relacionados con el rendimiento académico tales como el género, el estrato socioeconómico, el ciclo educativo, las tecnologías de información, modelo de educación a distancia vs presencial, entre otros.

\section{CONCLUSIONES}

En este artículo, se identifica por medio de una serie de técnicas inteligentes, los principales factores influyentes en el rendimiento académico de un estudiante. Los resultados permitieron establecer que los factores más influyentes en el desempeño académico son la pedagogía de los profesores, los horarios de clase adecuados, la buena relación docente-estudiante, la calidad académica de los docentes y un poco de actividades de trabajo extracurricular. Esto se logró con base en el algoritmo de clasificación bayesiano J48, 
el cual permite generar un árbol con todo el conjunto de posibles atributos influyentes en el desempeño académico de un estudiante. En general, las técnicas aquí empleadas permitieron lograr una efectividad del $91.67 \%$. No obstante, es necesario avanzar en nuevos estudios que permitan observar resultados considerando el nivel de formación (pregrado, maestría, doctorado), la región/país en dónde se ubica la institución, incluyendo otros factores que pudieran ser influyentes en el desempeño académico de los estudiantes.

\section{AGRADECIMIENTOS}

Se agradece la colaboración al Universidad Nacional de Colombia - Sede Manizales por el apoyo prestado en la realización de esta investigación.

\section{REFERENCIAS}

Alberola, J. y E. Val, An artificial intelligence tool for heterogeneous team formation in the classroom, http://dx.doi.org/10.1016/j.knosys.2016.02.010, Knowledge-Based Systems, 101, 1-14 (2016)

Barton, M. y I. Cohen, How is gentrification associated with changes in the academic performance of neighborhood schools?, https://doi.org/10.1016/j.ssresearch.2019.01.005, Social Science Research, 80, 230 - 242 (2019)

Bravo M., S. Salvo y C. Muñoz, Profiles of Chilean students according to academic performance inmathematics: An exploratory study using classification trees and random forests, http://dx.doi.org/10.1016/j.stueduc.2015.01.002, Studies in Educational Evaluation, 44, 50-59 (2015)

Castrillon, O., W. Sarache y E. Castaño, Sistema Bayesiano para la predicción de la diabetes, http://dx.doi.org/10.4067/S0718-07642017000600017, Información Tecnológica, 28(6), 161-168 (2017)

Castrillón, O., J. Giraldo y S. Ruiz, Principales factores influyentes en el rendimiento académico: Un caso de estudio, http://www.laccei.org/LACCEI2018-Lima/work_in_progress/WP207.pdf, 16th LACCEI International Multi-Conference for Engineering, Education, and Technology: "Innovation in Education and Inclusion", 19-21, Lima, Perú (2018)

Çilan, C.A. y M. Can, Measuring Factors Effecting MBA Students' Academic Performance by Using Categorical Regression Analysis: A Case Study of Institution of Business Economics, Istanbul University, doi: 10.1016/j.sbspro.2014.01.1362, Procedia - Social and Behavioral Sciences, 122, 405 - 409 (2014)

Crişan, C. y L. Copaci, The Relationship between Primary School Childrens' Test Anxiety and Academic Performance, doi: 10.1016/j.sbspro.2015.02.311, Procedia Social and Behavioral Sciences, 180, 1584 - 1589 (2015)

D'Alessio, F.A., B.E. Avolio y V. Charles, Studying the impact of critical thinking on the academic performance of executive MBA students, https://doi.org/10.1016/j.tsc.2019.02.002, Thinking Skills and Creativity, 31, 275-283 (2019)

De-La-Hoz, E.J., E.J. De-La-Hoz y T.J. Fontalvo, Metodología de Aprendizaje Automático para la Clasificación y Predicción de Usuarios en Ambientes Virtuales de Educación, http://dx.doi.org/10.4067/S0718-07642019000100247, Información tecnológica, 30(1), 247-254 (2019)

Etxeberria Paulo, A. Elisabete, y otros dos autores más, Análisis del Rendimiento Académico en Relación al Perfil de Ingreso del Alumnado e Identificación de Carencias Formativas en Materias Básicas de dos Grados de Ingeniería, doi: 10.4067/S0718-50062017000400007, Formación Universitaria, 10(4), 67-74 (2017)

Fernandes, E., M. Holanda y otros 4 autores, Educational data mining: Predictive analysis of academic performance of public school students in the capital of Brazil, https://doi.org/10.1016/j.jbusres.2018.02.012, Journal of Business Research, $94,335-343(2019)$

Fonteyne, L., W. Duyck y F. De Fruyt, Program-specific prediction of academic achievement on the basis of cognitive and non-cognitive factors, http://dx.doi.org/10.1016/j.lindif.2017.05.003, Learning and Individual Differences, 56, 34-48 (2017)

Garbanzo, G.M., Factores asociados al rendimiento académico en estudiantes universitarios, una reflexión desde la calidad de la educación superior pública, https://www.redalyc.org/articulo.oa?id=44031103, Revista educación, 3(1), 4363 (2007)

Hamsa, H., S. Indiradevi y J.J. Kishakkethottam, Student academic perfomance prediction model using decision tree and fuzy genetic algorithm, doi: 10.1016/j.protcy.2016.08.114, Procedia Technology, 25, 326 - 332 (2016)

Hejazi, E., Z. Naghsh y otros dos autores, Prediction of academic performance: the role of perception of the class structure, motivation and cognitive variables, doi:10.1016/j.sbspro.2011.04.054, Procedia Social and Behavioral Sciences, 15, 2063 - 2067 (2011)

Helal, S., J. Li y otros cinco autores, Predicting academic performance by considering student heterogeneity, https://doi.org/10.1016/j.knosys.2018.07.042, Knowledge-Based Systems, 161, 134-146 (2018)

Kweon, B.S., C.D. Ellis y otros dos autores, The link between school environments and student academic performance, http://dx.doi.org/10.1016/j.ufug.2017.02.002, Urban Forestry \& Urban Greening, 23, 35-43 (2017) 
Li, X. y M. de Rijke, Characterizing and predicting download in academic search, https://doi.org/10.1016/j.ipm.2018.10.019, Information Processing and Management, 56, 394-407 (2019)

Mandelman, S., B. Barbot y E.L. Grigorenko, Predicting academic performance and trajectories from a measure of successful intelligence, http://dx.doi.org/10.1016/j.lindif.2015.02.003, Learning and Individual Differences, 51, 387-393 (2016)

Miguéis, V.L., A. Freitas y otros dos autores más, Early segmentation of students according to their academic performance: A predictive modelling approach, https://doi.org/10.1016/j.dss.2018.09.001, Decision Support Systems, 115, 36-51 (2018)

Mitrofan, N. y A. Ion, Predictors of Academic Performance. The Relation between the Big Five Factors and Academic Performance, doi: 10.1016/j.sbspro.2013.04.264, Procedia - Social and Behavioral Sciences, 78, 125 - 129 (2013)

Pandey, M. y S. Taruna, Towards the integration of multipleclassifier pertaining to the Student'sperformance prediction, http://dx.doi.org/10.1016/j.pisc.2016.04.076, Perspectives in Science, 8, 364 - 366 (2016)

Paunonen, S. y M. Ashton, On the prediction of academics performance with personality traits: A replication study, https://doi.org/10.1016/j.jrp.2013.08.003, Journal of research in personality, 47(6), $778-781$ (2013)

Rendón R., La dinámica escolar: de la diferencia a la desigualdad, DOI: 10.5354/0719-0581.2012.18656, Revista de Psicología, (6), 7-18 (1997)

Secretaria de Educación Distrital de Bogotá, Estudio sobre los factores que influyen en el rendimiento Escolar, Tomado de.http://evaluacion.educacionbogota.edu.co/files/Factores\%20que\%20influyen\%20en\%20el\%20rendimiento\%20escolar .pdf, (2010)

Taylan, O. y B. Karagozog `lu, An adaptive neuro-fuzzy model for prediction of student's academic performance, doi:10.1016/j.cie.2009.01.019, Computers \& Industrial Engineering, 57, 732-741 (2009)

Valdivieso, C.E., R. Valdivieso y O.A. Valdivieso, Determinación del tamaño muestral mediante el uso de árboles de decision, DOI: 10.23881/idupbo.011.1-4e , UPB - Investigación \& Desarrollo, 11, 148 - 176 (2011)

Valencia, M., J. Correa y F. Díaz, Métodos estadísticos clásicos y bayesianos para el pronóstico de demanda. Un análisis comparativo, https://doi.org/10.15446/rev.fac.cienc.v4n1.49775, Revista Facultad de Ciencias Universidad Nacional de Colombia, 4(1), 52 -67 (2015)

Vedel A., D. Thomsen y L. Larsen, Personality, academic majors and performance: Revealing complex patterns, http://dx.doi.org/10.1016/j.paid.2015.04.030, Personality and Individual Differences, 85, 69-76 (2015)

Yousif, M.K. y A. Shaout, Fuzzy logic computational model for performance evaluation of Sudanese Universities and academic staff, http://dx.doi.org/10.1016/j.jksuci.2016.08.002, Journal of King Saud University Computer and InformationSciences, Computer and Information Sciences, 30, 80-119 (2018)

Zhu, D., L. Hodgkinson y Q. Wang, Academic performance and financial forecasting performance: $A$ survey study, https://doi.org/10.1016/j.jbef.2018.07.002, Journal of Behavioral and Experimental Finance, 20 , 45-51 (2018) 